\title{
Analysis of Abdominal ECG Signal for Fetal Heart Rate Estimation Using Adaptive Filtering Technique
}

\author{
Ashraf Adamu Ahmad, Aminu Inuwa Kuta \\ Dept. of Electrical and Electronic Engineering, Federal University of Technology, Minna, Nigeria \\ Email: a.ashraf@futminna.edu.ng, aik_kuta@yahoo.com \\ Abdulmumini Zubairu Loko \\ Dept. of Physics/Electronics, Nassarawa State University, Keffi, Nigeria \\ Email:azubairuloko@yahoo.com
}

\begin{abstract}
This paper presents a method for fetal heart rate estimation from an abdominal electrocardiogram (ECG) signal based on adaptive filter analysis using least mean square (LMS) adaptive filtering algorithm in order to determine the health status of a baby in its mother's womb. The fetal ECG signal is extracted from abdominal ECG containing other sources of interference using the maternal ECG signal obtained from mother's chest cavity as the reference signal. Interference/noise model used for this work include the power-line noise, the white noise and the unwanted propagating maternal ECG signal. Thereafter, the heart rate is estimated using an automated peak voltage measurement algorithm at 75 percent threshold voltage. It is found that irrespective of the estimated heart rate of the baby, 100 percent estimation is achieved at signal-to-noise ratio (SNR) greater than or equal to $-31 \mathrm{~dB}$.
\end{abstract}

Index Terms - Fetal heart rate, electrocardiogram (ECG), least mean square (LMS), power-line noise, white noise, signal-to-noise ratio (SNR).

\section{INTRODUCTION}

Fetal heart rate estimation is an aspect of fetal heart rate monitoring associated with estimating the heart rate of a baby from a pregnant mother abdominal electrocardiogram (ECG) signal measured at the womb in order to determine the health status of the baby. The status of the baby may either be normal within average heart rate or in distress with a low or excessively high heart rate or pathologic condition such as asphyxia [1]. Thereafter further investigations may be carried out on the baby in distress in order to determine the reason for the abnormal heart rate. Another problem is the extracted fetal ECG signal which is always marred with various sources of noise and interferences. These sources include the ones from the ECG device (power-line and white noise) and the mother herself (propagating maternal ECG signal) among other sources [2][3]. There is therefore the need to use appropriate signal processing techniques for accurate ECG signal analysis.

These techniques include fundamental Fourier transform [4], wavelet transform [5][6], support vector machine [7], robust tensor decomposition and extended kalman filtering [8], spatial filtering and adaptive rule [9], principal component analysis [10] among many others. Each of these methods is associated with its performance and accompanying limitations. Recently, combination of linear discriminator analysis (LDA) and cross correlation was used to detect different human states from multiple ECG signals with accuracy of 90-95 percent [11], Mestimation methods (a weight model) for fetal heart rate estimation in impulse noises [12], and adaptive noise cancellation and digital filter for fetal ECG extraction [13]. A comprehensive review of ECG signal processing methods is presented in [14]

This work identifies the adaptive filtering technique as a key signal processing tool for heart rate estimation at a very good SNR. This technique uses an adaptive algorithm with a reference desired signal so that the output can match this signal after various iterative processes leaving the error/unmatched signal at the end. In this work, the least mean squares (LMS) method is used as the adaptive algorithm. The maternal ECG signal obtained at the probe of the mother's chest cavity is used as the reference signal to the input abdominal ECG signal obtained at the probe of the womb. Recently this technique was used for acoustic echo cancellation [15], GPS multipath error estimation and mitigation [16] and estimation of hyper-complex quantity (rotor) able to describe rotations in any dimension [17].

Other sections of the paper is organized as follows: section II present the abdominal ECG signal model, section III discuss analysis and method of the algorithm used, finally results obtained and discussions accompanying them are presented in section IV followed by a concluding section..

\section{ABdominal ECG SignAL MODEL}

The abdominal ECG signal $(A(n))$ obtained from the 
mother's womb is a combination of various ECG signals and noises. The fetal ECG signal $(F(n))$ is the main ECG signal present at the womb of typically a low signal power of $0.25 \mathrm{mV}$ and of normal heart rate of between $110 \mathrm{bpm}$ and $160 \mathrm{bpm}$ [3]. The other present ECG signal is the maternal ECG signal $(M(n))$ measured from the mother's chest cavity which is used as a reference signal for the adaptive filtering technique and undergoes a nonlinear propagation to the mother's abdomen. Its signal power is typically of $3.5 \mathrm{mV}$ and also a typical heart rate of $89 \mathrm{bpm}[18]$. The sampling frequency in this work is $4000 \mathrm{~Hz}$ based on standarddesktop ECG devices [19],which facilitates the conversion of the ECG signal into samples for digital processing. The two ECG signals are graphically depicted in Fig. 1.

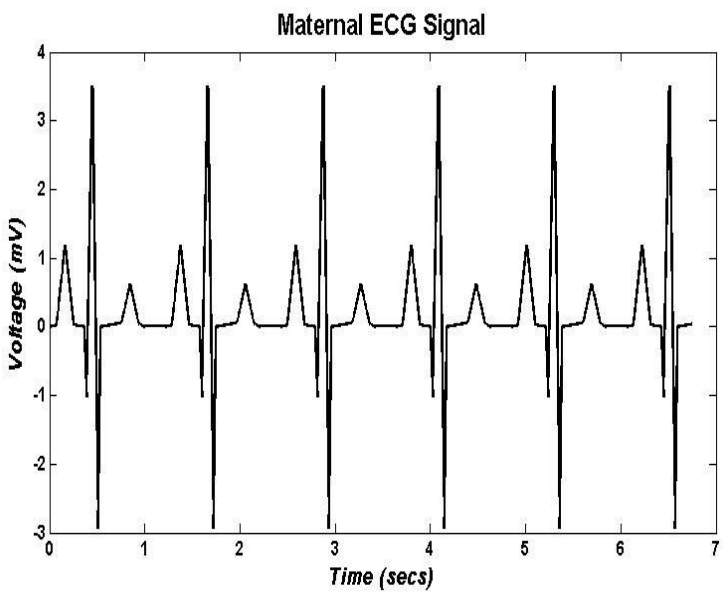

(a)

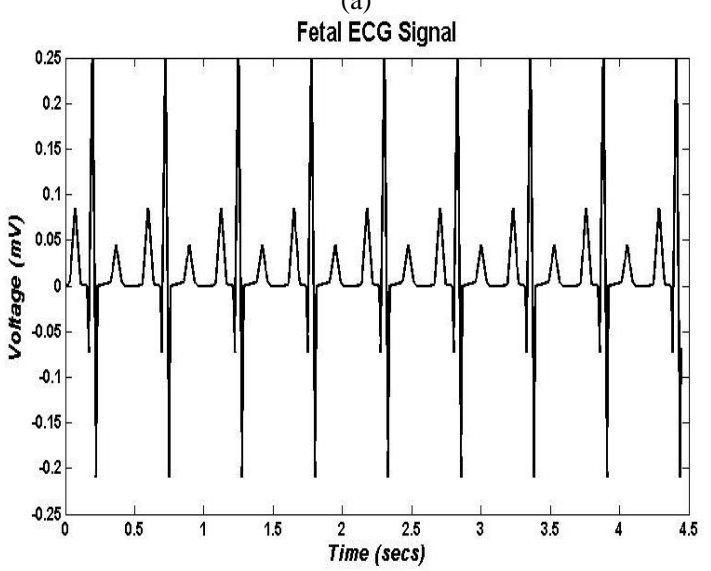

(b)

Fig.1. Graphical depiction of two main ECG signals (a)Maternal ECG signal (b) Fetal ECG Signal

Fig. 1 shows the low power of the fetal ECG signal as compared to that of the maternal ECG signal. Also it is seen that more heartbeats are captured in the fetal signal than that of the maternal signal due to its higher heart rate. In this work, any signal apart from the fetal ECG $(F(n))$ at the abdomen is considered to be noise. This noise is composed of three parts; the power-line noise $(P(n))$, the white noise $(W(n))$ and the maternal signal propagation from the chest cavity to the abdomen $(M(n))$. The simulation set-up of the abdominal ECG signal used in this work is shown in Fig. 2.

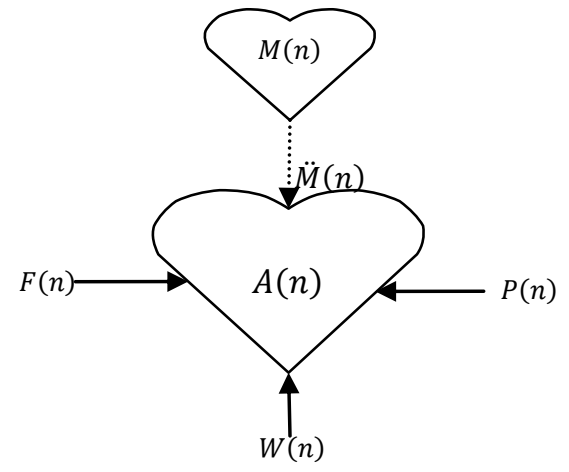

Fig.2. Pictorial depiction of the abdominal ECG signal model

The maternal signal arrow shown in Fig. 2 is given in dotted format in order to show the non-linear propagation from the chest cavity to the abdomen as opposed to others that are constituents of the abdominal ECG signal. The power-line noise originates from the power source an ECG measuring device and it is modeled by a sinusoidal equation shown in equation 1 .

$$
P(n)=A \sin 2 \pi f / f_{s}(1: n)
$$

Where $P$ is the power line noise, $A$ is the signal power, $f$ is the frequency, $f_{s}$ is the sampling frequency and $1: n$ represents the duration of signal (same length as that of the abdominal). Signal power of $0.001 \mathrm{mV}$ and frequency of $50 \mathrm{~Hz}$ selected based on national power supply frequency. The white noise is the additive white Gaussian noise (AWGN) of Gaussian probability density function (equation 2), impulse response autocorrelation (equation 3 ) and all frequency power spectrum (equation 4)[20];

$$
\begin{gathered}
P_{x}(x)=\frac{1}{\sigma \sqrt{2 \pi}} e^{-(x-\bar{x})^{2} / 2 \sigma^{2}} \\
R_{x x}(\tau)=K \delta(\tau) \\
S_{x x}(w)=K
\end{gathered}
$$

Where $\bar{x}$ is the mean, $\sigma^{2}$ is the variance and $K$ is a constant.The white noise is obtained by generation of normally distributed pseudorandom numbers of standard mean of zero and variance corresponding to the signal power of the noise. There is also presence of maternal ECG signal in the abdomen as a result of non-linear of propagation from the chest cavity to the abdomen. This non-linear propagation is modeled by a linear FIR filter of ten randomized coefficients with formula shown in equation (5) [21];

$$
\ddot{M}(n)=\sum_{k=0}^{N} b(k) M(n-N)
$$

Where $b$ denotes the ten randomized filter coefficients obtained from the hamming window used in this work. The total noise can therefore be given as;

$$
N(n)=P(n)+W(n)+\ddot{M}(n)
$$


Henceforth, a graphical depiction of an abdominal ECG signal at a signal-to-noise ratio (SNR) of $-18 \mathrm{~dB}$ is shown in Fig. 3.

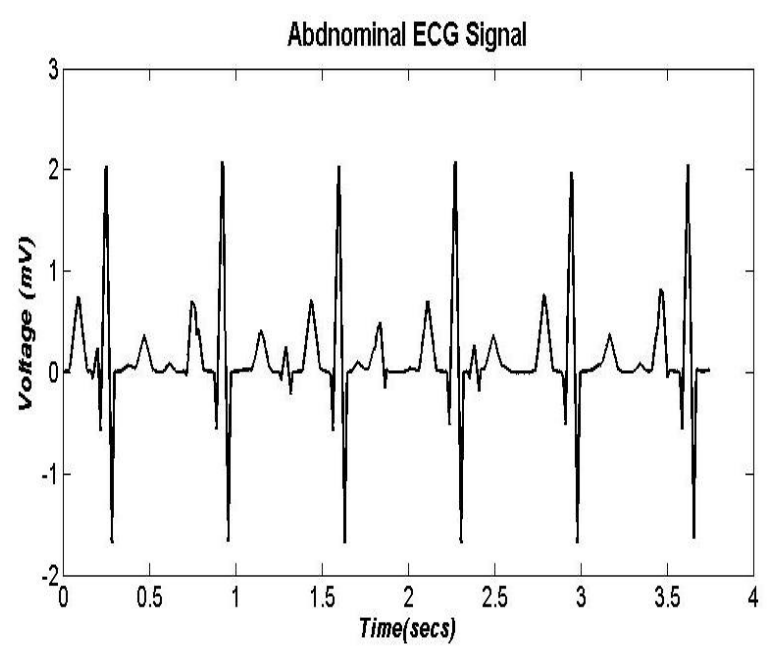

Fig.3. Abdominal ECG signal at SNR of -18dB.

It is seen that in Fig. 3, it is quite hard to differentiate the various ECG signals present in the signal. This is even more so when the effect of noise is higher.

\section{AdAPTIVE SignAl FILTERING TECHNIQUE}

The term filter can be used to describe a system of whose sole purpose is to extract information of interest from noisy data. This is because in the real world, the output signal (the received signal) is always in a distorted form of the input signal (the transmitted signal) after passing through the channel (processing system). As such a system or the estimator is then required in order to obtain original input from the output using prior information. This process is depicted in the block diagram of Fig. 4.

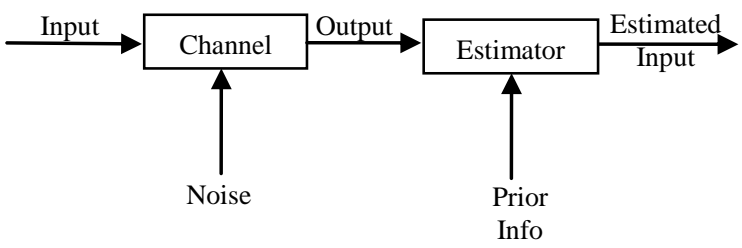

Fig.4. Block diagram depicting filter theory

At the end of the whole process shown in Fig.4, a form of originally sent input is estimated. The closeness of this estimated input to the originally sent input would depend on quality of the estimator system and the field of interest. These fields of interest include radar, sonar, communications, and biomedical engineering among many others. The estimator used in this work is the adaptive filter technique.

Time variable filters uses multiplier with time-variable coefficients so as that its characteristics can be varied with time. This filter uses an adaptation mechanism by which these multiplier coefficients can be adjusted so as to achieve optimum performance. This type of filter is called an adaptive filter [22]. The instantaneous values of the multiplier coefficients are evaluated through an incorporated optimized algorithm in the adaptation mechanism such that some norm of an error function of equation (7) is minimized.

$$
e(n)=d(n)-y(n)
$$

Where $d(n)$ is some desired reference signal and $y(n)$ is the filter output. A typical adaptive filter configuration is given in Fig. 5.

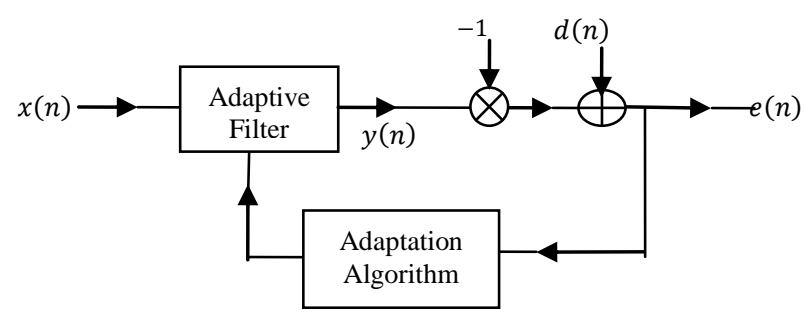

Fig.5. Adaptive filtering technique

The -1 input in Fig. 5 represents the delay in the input signal required for the adaptive filtering in order to improve on its previous output. The error signal $(e(n))$ is fed into to the adaptive filter through the adaptation algorithm at various increments of time, n. Over this time, output of the adaptive filter becomes a better and better match to the desired response signal through the adaptation process such that the magnitudes of error signal $(e(n))$ decreases over time as matched to this desired response signal [23].

In this work, the least mean squares (LMS) adaptation algorithm is used for the fetal heart rate estimation based on. There are various LMS based finite impulse response (FIR) adaptive filter algorithms such as the adjoint, block, fast Fourier transform (FFT) based block, filtered-X among many others [24], but in this work, the basic form is used. Its formula is gotten from an update of the steepest-descent algorithm given in equation (8);

$$
a(n+1)=a(n)+2 \alpha e(n) x(n)
$$

Where $a$ represents the filter coefficients $\alpha$ represents the convergence within the range given in equation (9) in order to ensure the convergence of the algorithm;

$$
0<\alpha<\frac{1}{N E\left[x^{2}(n)\right]}
$$

$N$ is the order of the filter, $E$ is the expectation operator and $E\left[x^{2}(n)\right]$ represents the average input power. The signal flow graph representation of the LMS algorithm is shown in Fig. 6 [23]. 


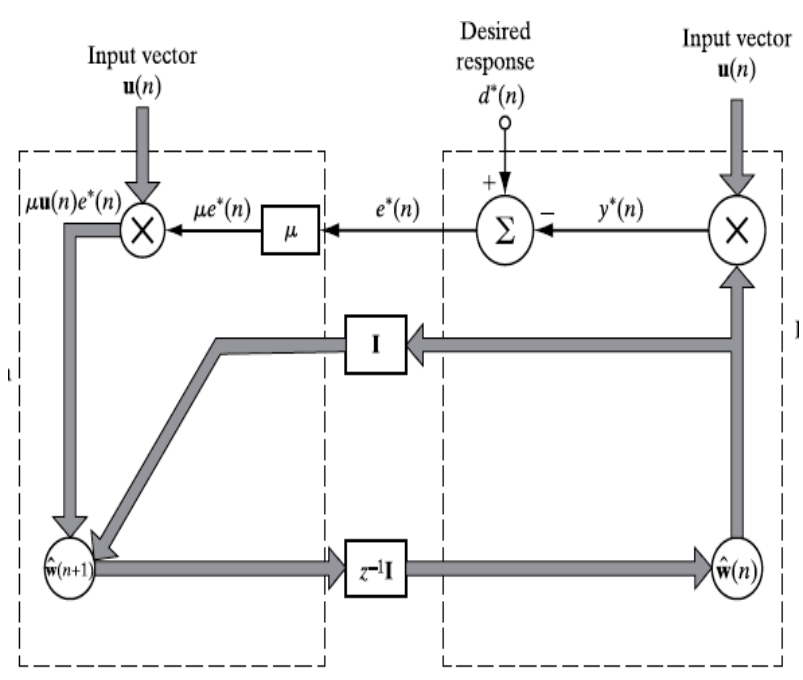

Fig.6. Signal flow graph representation of LMS algorithm

$\mathrm{I}$ is the identity matrix, $\mathrm{z}^{-1}$ is the unit delay operator and $\mu$ is the step-size parameter. It is seen that the signal flow graph in Fig. 6 is divided into two parts; the right hand side represents the filtering process responsible for producing the output and the error while the left hand side represents the adaptation process responsible for updating the weight vector of the LMS. When the aforementioned model of Fig. 5 is applied to the objective of this work, the adaptive filtering model gotten is shown in Fig. 7.

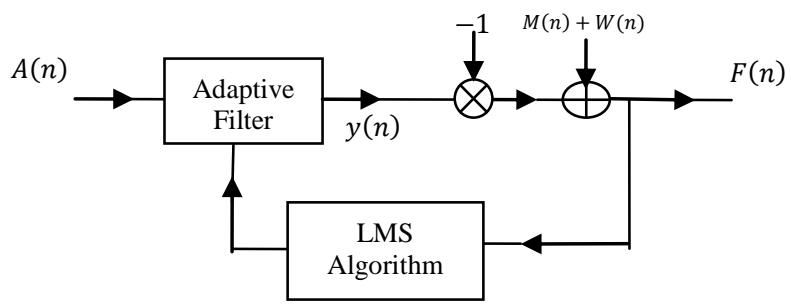

Fig.7. Adaptive filtering technique for fetal heart rate estimation

It is seen from Fig. 7, that over increment of time, the output $(y(n))$ would try to its possible best to match the desired signal $M(n)+W(n)) \cdot W(n)$ is the small amount of white noise added to the maternal signal obtained at the chest cavity in order to cater for the measurement noise. At the end of the adaptive process, the signal of interest, the error signal which is also the fetal ECG signal is obtained. A positive integer of 10 was used for the number of coefficients, the step size of 0.001 , and default leakage factor of 1.0 was used for the LMS adaptive filter in this fetal heart rate estimation set up.

From the obtained fetal ECG signal, heart rate of the baby (the fetal heart rate) is estimated from the interval between the first and second peak of the obtained signal using a 75 percent threshold. The threshold is selected in this way so as to cater for the noise associated with the extracted fetal ECG signal. Thereafter, the obtained heart rate in samples is converted and round to the nearest figure in bpm using the sampling frequency.

\section{RESULTS AND DISCUSSION}

Test fetal heart rates in bpm of 55, 110, 135, 160 and 320 are used to verify the performance of the adaptive signal processing technique for various signal-to-noise ratios (SNRs). Heart rates of 110-160bpm represents the standard heart rates of a healthy baby while that of $55 \mathrm{bpm}$ and $320 \mathrm{bpm}$ is used to represents two extremes of a baby in distress, with one representing half the minimum acceptable heart rate and the other twice the maximum acceptable heart rate. The SNR in decibels is given by equation 10 .

$$
\operatorname{SNR}(d b)=10 \log _{10} \frac{P_{S}}{P_{N}}
$$

Where $P_{S}$ is the signal power, and $P_{N}$ is the noise power. Different SNRs are obtained by varying the noise power; the results obtained are shown in Fig. 8.

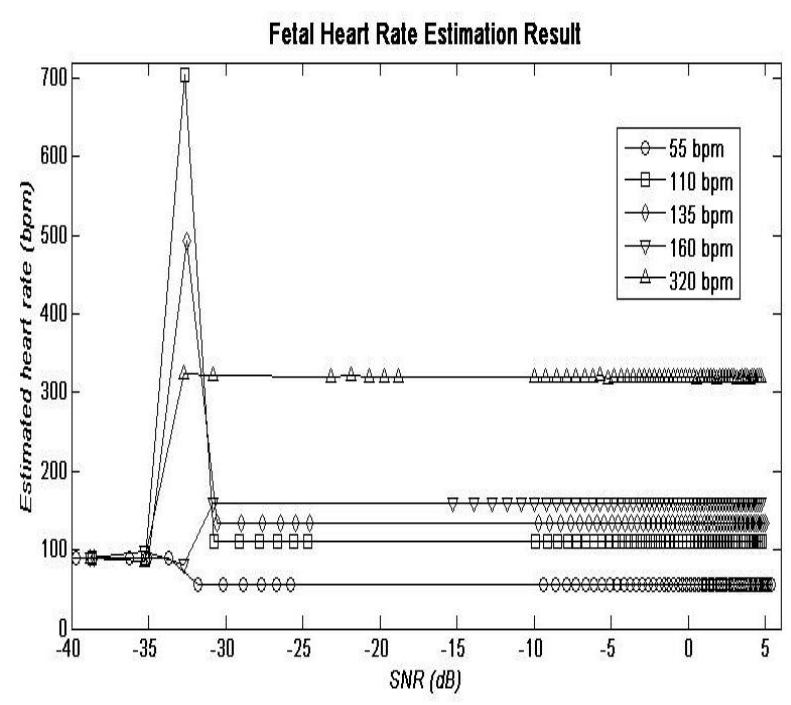

Fig.8. Results obtained for various heart rates

From Fig.8, the heart rate is wrong and unpredictable at SNR less than $-31 \mathrm{~dB}$ irrespective of the heart rate in consideration, therefore the SNR of $-31 \mathrm{~dB}$ is seen as the optimum SNR for the fetal heart rate estimation using the LMS adaptive filtering technique. This is further seen in Table 1 where the optimum SNR for the estimation of each heart rate considered is shown. The spikes in Fig.8are due to unpredictable nature of the method used when the noise is very high and mostly associated with the automatic voltage measurement algorithm using threshold just like in [25][26].

In these aforementioned papers, inter-pulse analysis of radar signals are carried out to determine the time characteristics of incoming emitter signal using smoothed instantaneous energy [25] and the short time Fourier transform [26]. Unlike this work, these methods used 50 percent threshold as opposed to the 75 percent used due to the differing nature of the noise environment, signal of interest and simulation set-up. The noise environment in those papers is modeled by a single environmental source of additive white Gaussian noise (AWGN) due to the microwave environment used for radar signals. The 
signal of interests included low probability of intercept signal (LPI) signals of low power characteristics and nonsinusoidal modulation while the simulation set-up was based on practical implication of time and frequency parameters.

Table 1. Optimum SNR for various fetal heart rate estimations

\begin{tabular}{|c|c|}
\hline Heart rate $(\mathrm{bpm})$ & SNR $(\mathrm{dB})$ \\
\hline 55 & -32 \\
\hline 110 & -31 \\
\hline 135 & -31 \\
\hline 160 & -31 \\
\hline 320 & -33 \\
\hline
\end{tabular}

Further analysis of table 1 shows that optimum SNR for normal heart rate of healthy baby at $-31 \mathrm{~dB}$ is higher than that of the abnormal rate of $-32 \mathrm{~dB}$ and $-33 \mathrm{~dB}$, requiring increment $\mathrm{SNR}$ of $1 \mathrm{~dB}$ or $2 \mathrm{~dB}$. This can be attributed to two reasons. Firstly, the LMS based adaptive filter and the heart rate estimation performance algorithms are iterative in nature. Their iterative nature ensures a form of unpredictability. Secondly and more importantly, the threshold of 75 percent was selected in order to put the normal heart rate of healthy baby in view.

Superiority of the algorithm used can also be demonstrated by comparing the extracted fetal ECG signal obtained from the abdominal ECG signal at the optimum SNR result which the noise power is much more than signal power and at a SNR for which the signal power is much more than the noise power. The earlier case of the extracted fetal ECG signal is shown in Fig. 9.

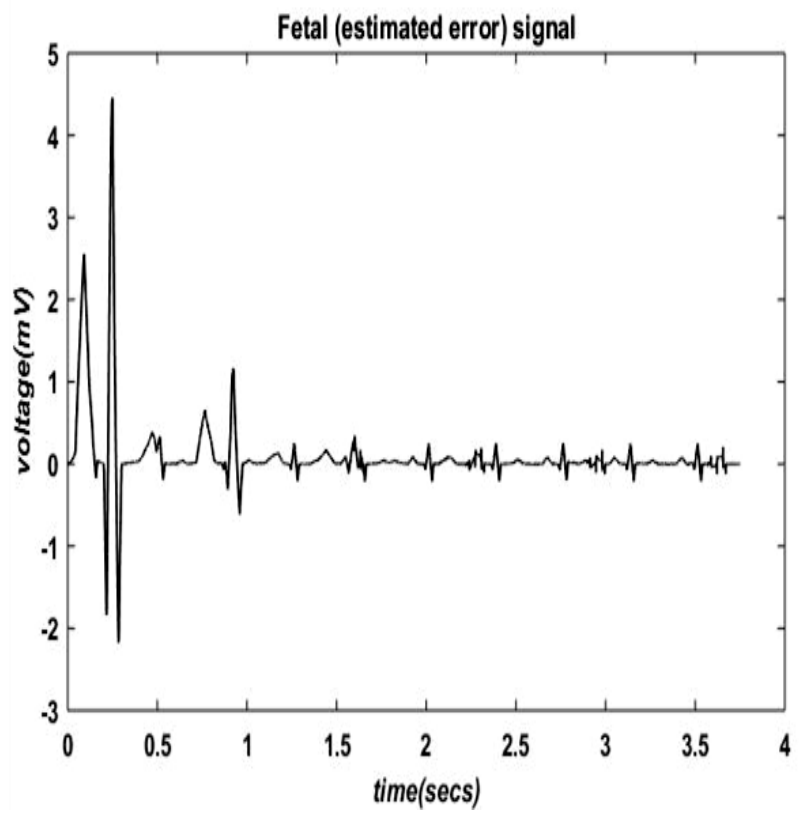

Fig.9. Extracted fetal ECG signal at SNR of $-31 \mathrm{~dB}$

It takes at least $1 \mathrm{sec}$ to obtain an extracted fetal ECG signal of its original standard low power of $0.25 \mathrm{mV}$ as shown in Fig. 9 due to required convergence of the LMS algorithm in the presence of high level of noise. It is also seen that ECG components are also non-homogenous as compared to the standard fetal ECG signal for the same reason. This is illustrated in a capture of 0.5 secs (between 1.2 secs to 1.7 secs) shown in fig. 10 .

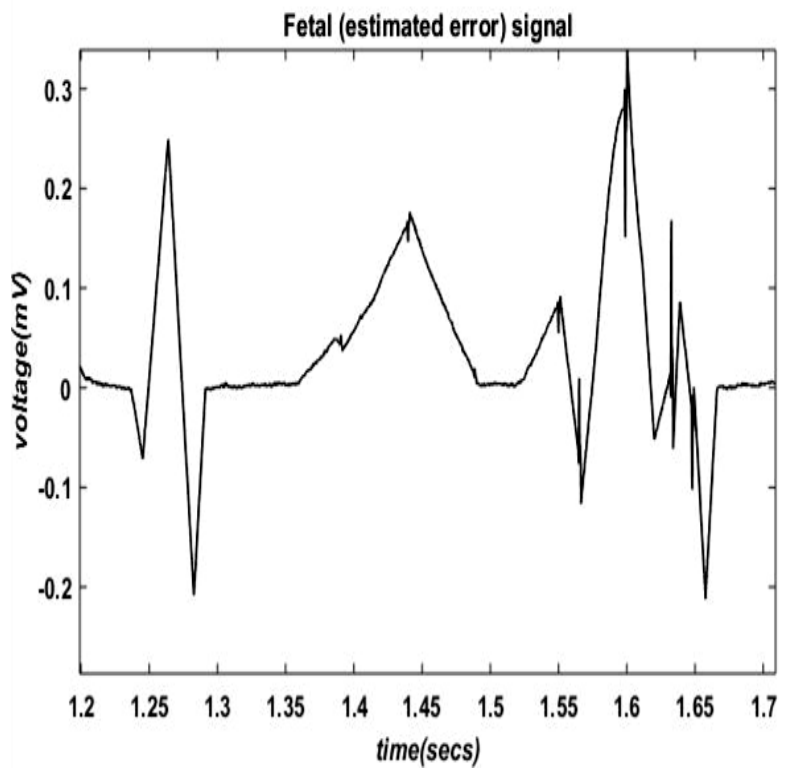

Fig.10. Capture of extracted fetal ECG signal at SNR of -31dB

Despite these shortcomings as depicted in fig. 9 and fig. 10 , the used method is still able to accurately estimate the heart rate from the peaks of the extracted fetal ECG. At a much better SNR, the shortcomings of high power and non-homogenous of the fetal ECG signal are absent as it is marred by noise of lower power. This is further seen in fig. 11 .

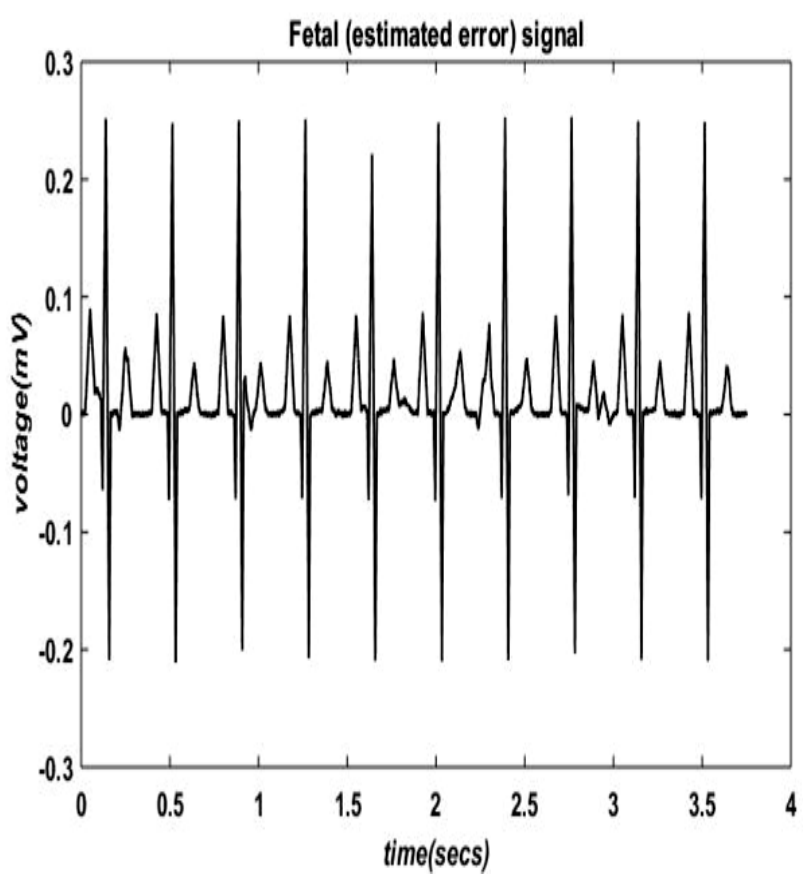

(a) 


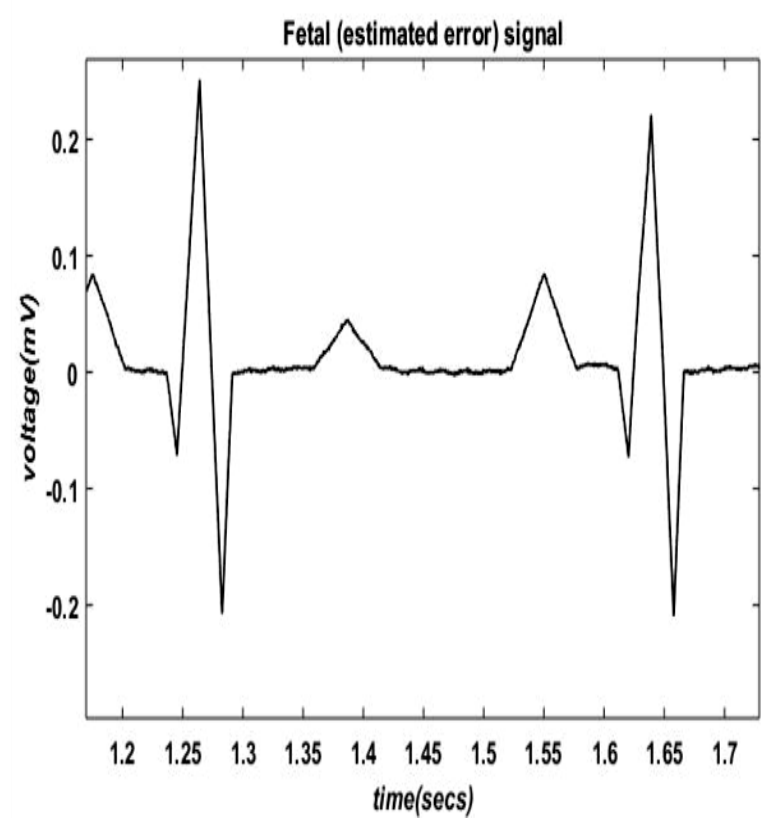

(b)

Fig.11. Extracted fetal ECG signal at SNR of 10dB (a) Full ECG signal (b) $0.5 \mathrm{sec}$ capture of the ECG Signal

At this good SNR in fig. 11, the method used estimate the heart rate accurately as expected due to the favorable noise condition. Also, the good performance at very low SNR also shows the strength of the adaptive signal processing technique used in this work and can also be attributed to the high sampling frequency when compared to the signal center frequency (heart rate). This is unlike other fields such as those of radar or wireless where same luxury is not possible based on current available technologies[27][28]. This can be deduced by using the frequency resolution formula in analog format shown in equation 11 .

$$
R_{f}=F_{s} / F_{c}
$$

Where $F_{S}$ is the sampling frequency and $F_{c}$ is the center frequency (replacing the sampling points $N$ for the sake of this discussion).The maximum allowed heart rate of a healthy baby of $160 \mathrm{bpm}$ translates to a center frequency of $3 \mathrm{~Hz}$ (or 3bps) approximately, and with a sampling frequency of current desktop ECG device of $4000 \mathrm{~Hz}$ gives a frequency resolution of approximately 1333. In radar technology, the center (intermediate) frequency of the basic radar signal is $10 \mathrm{MHz}$, and with a sampling frequency of $80 \mathrm{MHz}$ based on current radar system, gives a frequency resolution of just 8 . It is quite clear why the performance of this work is very good. However, it is important that the sampling frequencies of handheld ECG devices are much lower due to the miniaturization.

Discussion of results cannot be complete without comparing results obtained in this paper with that of previous similar works and as such three closely related works were identified among many others. This other works (such as the ones mentioned in the introductory section) use different methods and more importantly quite different performance ratio, making it harder and unfair to compare with this work. In [29], performances of various window techniques for ECG signal noise cancellation were evaluated. AWGN was added artificially to ECG signals obtained from MIT-BIH database and three window based FIR filters were considered; Gaussian, Bartlett and Von Hann. The SNR obtained after filtering are shown in Table 2.

Table 2. SNR of window techniques for ECG noise cancellation

\begin{tabular}{|l|l|}
\hline Window function & Performance Measure \\
\hline Bartlett Window & -3.7648 \\
\hline Gaussian Window & -3.4710 \\
\hline Von Hann Window & -3.7082 \\
\hline
\end{tabular}

Table 2 shows that the Bartlett is the best, followed by Von Hann with Gaussian coming last among the windows considered in the work. In a very similar set-up to this paper, a combination of LMS based adaptive filter and genetic algorithm optimization technique is used for ECG extraction [30], however the input signal is also obtained from the MIT-BIH database. This probably accounts for poorer performance of $-11.2 \mathrm{~dB}$ as compared to this work of $-31 \mathrm{~dB}$ despite the superiority of the method used. In the third paper [31], wavelet analysis and fuzzy thresholding is used to filter weak ECG signals corrupted by AWGN. The wavelet analysis is used for decomposition and reconstruction while fuzzy s-function determines the threshold. Notable denoising is achieved at SNR lesser than $-20.6 \mathrm{~dB}$.

Summary of these results obtained in this work compared with other aforementioned fetal heart rate analysis methods are shown in Table 3. Each of these methods has some slight differences in the methodology and experimental set ups but with the main similarity focusing on ECG signal de-noising and noise environment considered.

Table 3. Various fetal heart rate analysis methods

\begin{tabular}{|l|l|}
\hline Method & $\begin{array}{l}\text { Optimum } \\
\text { SNR }\end{array}$ \\
\hline Window Technique [29] & -3.8 \\
\hline GA Based Adaptive Filtering [30] & -11.2 \\
\hline Wavelet Analysis and Fuzzy Thresholding [31] & -20.6 \\
\hline LMS Adaptive Filtering & -31 \\
\hline
\end{tabular}

It is seen in Table 3 that the LMS adaptive filtering method is superior to other presented methods. The next superior method is the wavelet analysis followed by genetic algorithm based (GA) based adaptive filtering. The window functions technique is the least superior due to its simplicity when compared to other methods presented in the table. It is however important to point out that the two main shortcoming of the method used in this work is associated with its dependence on the maternal signal measurement from mother's chest cavity for fetal heart rate estimation and also its fairly high computational complexity of the adaptive filter due to the iterative process as compared to other methods. 


\section{CONCLUSION}

This work presents a method of estimating fetal heart from extracted fetal ECG signal from the abdominal ECG signal with the aid of the basic LMS adaptive filtering technique. Various heart rates are estimated accurately at SNR of greater than or equal to $-31 \mathrm{~dB}$. Further investigation will involve the use of actual ECG signal and time-frequency analysis to track the spectral changes of various components of the abdominal ECG without the need to measure maternal ECG signal at the mother's chest cavity.

\section{REFERENCES}

[1] Rajesh, P., Umamaheswari, K. and Kumar, V.N., 2014. A Novel Approach of Fetal ECG Extraction Using Adaptive Filtering." International Journal of Information Science and Intelligent System, 3(2), pp.55-70.

[2] Nasiri, M., Faez, K., Nasrabadi, A.M., 2012. Fetal Electrocardiogram Signal Extraction by ANFIS Trained with PSO Method. International Journal of Electrical and Computer Engineering, 2(2), p.247.

[3] Ghodsi, M., Hassani, H. and Sanei, S., 2010. Extracting fetal heart signal from noisy maternal ECG by singular spectrum analysis. Journal of Statistics and its Interface, Special Issue on the Application of SSA, 3(3), pp.399-411.

[4] Lweesy, K., Fraiwan, L., Maier, C. and Dickhaus, H., 2009. Extraction of fetal heart rate and fetal heart rate variability from mother's ECG signal. World Academy of Science Engineering and Technology, 3, pp.599-604.

[5] Inan, O.T., Giovangrandi, L. and Kovacs, G.T., 2006. Robust neural-network-based classification of premature ventricular contractions using wavelet transform and timing interval features. Biomedical Engineering, IEEE Transactions on, 53(12), pp.2507-2515.

[6] Nilanjan Dey, Sayantan Mukhopadhyay,Achintya Das,Sheli Sinha Chaudhuri, "Analysis of P-QRS-T Components Modified by Blind Watermarking Technique Within the Electrocardiogram Signal for Authentication in Wireless Telecardiology Using DWT", International Journal of Image, Graphics and Signal Processing (IJIGSP), Vol.4, No.7, pp.33-46, 2012.

[7] Mehta, S.S. and Lingayat, N.S., 2007, May. Support Vector Machine for Cardiac Beat Detection in Single Lead Electrocardiogram. In IMECS (pp. 1630-1635).

[8] Akhbari, M., Niknazar, M., Jutten, C., Shamsollahi, M.B. and Rivet, B., 2013, September. Fetal electrocardiogram R-peak detection using robust tensor decomposition and extended kalman filtering. In Computing in Cardiology Conference (CinC), 2013 (pp. 189-192). IEEE.

[9] Xu-Wilson, M., Carlson, E., Cheng, L. and Vairavan, S., 2013, September. Spatial filtering and adaptive rule based fetal heart rate extraction from abdominal fetal ECG recordings. In Computing in Cardiology Conference (CinC), 2013 (pp. 197-200). IEEE.

[10] Petrolis, R. and Krisciukaitis, A., 2013, September. Multi stage principal component analysis based method for detection of fetal heart beats in abdominal ECGs. In Computing in Cardiology Conference (CinC), 2013 (pp. 301-304). IEEE.

[11] Shinwari, M.F., Ahmed, N., Humayun, H., ulHaq, I., Haider, S. and ulAnam, A., 2012. Classification algorithm for feature extraction using Linear Discriminant Analysis and Cross-Correlation on ECG signals. International Journal of Advanced Science and Technology, 48, pp.149-
162.

[12] Zeng, X., Zhou, Y., Tao, J., Yang, J. and Li, S., 2015. Mestimation Methods for Fetal Heart Rate Estimation in Impulse Noises. International Journal of Signal Processing, Image Processing and Pattern Recognition, 8(2), pp.175-184.

[13] Prasanth, K., Paul, B. and Balakrishnan, A.A., 2013. Fetal ECG Extraction Using Adaptive Filters. International Journal of Advanced Research in Electrical, Electronics and Instrumentation Engineering, 2(4), pp.1483-1487.

[14] Sameni, R. and Clifford, G.D., 2010. A review of fetal ECG signal processing; issues and promising directions. The open pacing, electrophysiology \& therapy journal, 3, p.4.

[15] KrishnaSamalla, G.Mallikarjuna Rao, Ch.Stayanarayana, "Survey of Sparse Adaptive Filters for Acoustic Echo Cancellation", International Journal of Image, Graphics and Signal Processing (IJIGSP), Vol.5, No.1, pp.16-24, 2013.DOI: 10.5815/ijigsp.2013.01.03.

[16] Swathi, N., Dutt, V.I. and Rao, G.S., 2016. An Adaptive Filter Approach for GPS Multipath Error Estimation and Mitigation. In Microelectronics, Electromagnetics and Telecommunications (pp. 539-546). Springer India.

[17] Lopes, W.B., Al-Nuaimi, A. and Lopes, C.G., 2016. Geometric-Algebra LMS Adaptive Filter and its Application to Rotation Estimation. Signal Processing Letters, IEEE, 23(6), pp.858-862

[18] Raj, J. and Sahu, A.K., 2014. Fetal electrocardiogram extraction and analysis (B.Tech dissertation). National Institute of Technology, Rourkela, India.

[19] Narayana, K.V.L. and Rao, A.B., 2011. Noise removal using adaptive noise canceling, analysis of ECG using Matlab. International Journal of Engineering Science and Technology, 3(4).

[20] Antoniou, A., 2006. Digital signal processing. Toronto, Canada:: McGraw-Hill.

[21] Widrow, B., Glover Jr, J.R., McCool, J.M., Kaunitz, J., Williams, C.S., Hearn, R.H., Zeidler, J.R., Dong Jr, E. and Goodlin, R.C., 1975. Adaptive noise cancelling: Principles and applications. Proceedings of the IEEE,63(12), pp.1692-1716.

[22] Haykin, S.S., 2008. Adaptive filter theory. Pearson Education India.

[23] Widrow, B. and Stearns, S.D., 1985. Adaptive signal processing. Englewood Cliffs, NJ, Prentice-Hall, Inc., 1985, 491 p., 1.

[24] Diniz, P.S.R., 2008. Adaptive Filtering: Algorithms and Practical Implementation. Springer. New York, NY, USA.

[25] Adam, A.A., Adegboye, B.A. and Ademoh, I.A., 2016. Inter-Pulse Analysis of Airborne Radar Signals using Smoothed Instantaneous Energy. International Journal of Signal Processing Systems (IJSPS). 4(2), pp.139-143.

[26] Ahmad, A.A., Daniyan, A. and Gabriel, D.O., 2015. Selection of Window for Inter-Pulse Analysis of Simple Pulsed Radar Signal using the Short Time Fourier Transform. International Journal of Engineering \& Technology, 4(4), pp.531-537.

[27] Ahmad, A.A. and Sha'ameri, A.Z., 2015. Classification of airborne radar signals based on pulse feature estimation using time-frequency analysis. Science \& technology research institute for defence, 8(2), p.103.

[28] Ahmad, A.A., Ayeni, J.B. and Kamal, S.M., 2015, September. Determination of the pulse repetition interval (PRI) agility of an incoming radar emitter signal using instantaneous power analysis. In AFRICON, 2015 (pp. 14). IEEE.

[29] Sharma, S. and Narwaria, R.P., 2014. Performance 
Evaluation of Various Window Techniques for Noise Cancellation from ECG Signal. International Journal of Computer Applications, 93(19).

[30] Kholdi, E., Bigdeli, N. and Afshar, K., 2011. A New GABased Adaptive filter for fetal ECG extraction. World Academy of Science, Engineering and Technology, 54.

[31] Üstündağ, M., Gökbulut, M., Şengür, A. and Ata, F., 2012. Denoising of weak ECG signals by using wavelet analysis and fuzzy thresholding.Network Modeling Analysis in Health Informatics and Bioinformatics, 1(4), pp.135-140.

\section{Authors' Profiles}

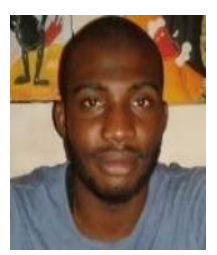

Ashraf A. Ahmad, is a Lecturer at the Federal University of Technology, Minna. He graduated from Bayero University, Kano in 2011 and UniversitiTeknologi Malaysia in 2014, obtaining B.Eng and M.Eng Electrical Engineering respectively. He received the best student award for his master's program. His research interests include digital signal processing and electronic intelligence. He has a number of publications both in international conferences and journals in the area of his interests.

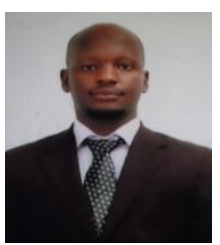

Aminu I. Kuta, is a Technologist at the Federal University of Technology, Minna. He graduated from Kaduna Polytechnic, Kaduna in 2006 and Bayero University, Kano in 2016, obtaining HND Electrical Engineering and M.Eng Control \& Instrumentation Engineering respectively. His research interests Include optimal control, power control and digital signal processing. He has a number of publications both in international conferences and journals in the area of interests.

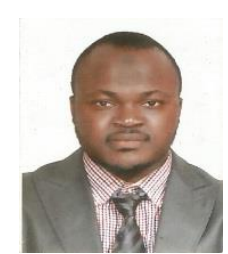

Abdulmumini Z. Loko,is a Lecturer at the Nasarawa State University, Keffi. He holds National Diploma in the year 2005, Higher National Diploma in the year 2008, from The Federal Polytechnic Nasarawa, Bachelor of Engineering from University of East London in the year 2012 and Masters of Engineering UniversitiTeknologi Malaysia in the year 2014. Before travelling Abroad for further studies, he had earlier obtained Bachelor of Science (B.Sc.) in Accounting from Nasarawa State University Keffi. His research interests include digital signal processing and Digital Electronic and embedded system. He has a number of publications, conferences and journals nationally and internationally in the area of his interests.

How to cite this paper: Ashraf Adamu Ahmad, Aminu Inuwa Kuta, Abdulmumini Zubairu Loko,"Analysis of Abdominal ECG Signal for Fetal Heart Rate Estimation Using Adaptive Filtering Technique", International Journal of Image, Graphics and Signal Processing(IJIGSP), Vol.9, No.2, pp.19-26, 2017.DOI: 10.5815/ijigsp.2017.02.03 